\title{
INFORMATIONS DIVERSES
}

The symposium on fibre-reinforced cement based composites will take place at Watford, U.K. The first meeting of the Organising Committee was held on 28th November under the chairmanship of Prof. A.M. Neville of University of Leeds who had just returned from a lecture tour in Canada. The symposium dates 15 to 17 September 1975 were confirmed and it was agreed that the programme should comprise an opening session on the morning of the 15th followed by five halfday technical sessions as follows :

\section{Sept. morning}

Opening session to include :

Welcome address

Keynote address

Short slide talks on products and applications.

\author{
15 Sept. Afternoon \\ Theoretical aspects of fibre reinfor- \\ cement of cement and concrete. \\ 16 Sept. morning \\ Properties and testing. \\ 16 Sept. afternoon \\ Optional industrial or laboratory visit. \\ 17 Sept. morning \\ Properties and testing.

\section{Sept. afternoon} \\ Applications.
}

Dr. A. Kelly, FRS, Deputy Director at National Physical Laboratory, is being invited to give the keynote address.
Un colloque sur la rhéologie des bétons, organisé par I'Association Française de Recherches et d'Essais sur les Matériaux et le Groupe Français de Rhéologie, s'est tenu à Paris les 10 et 11 décembre 1973.

Du 18 au 21 septembre 1973, à Prague un colloque RILEM/IUPAC sur la structure des pores et les propriétés des matériaux, a réuni environ 450 participants de 28 pays. Les sujets traités étaient regroupés sous 6 thèmes: modèles et géométrie de la structure des pores, formation de la structure des pores, méthodes de détermination de la structure des pores, relation entre la structure des pores et les propriétés mécaniques des matériaux, relation entre la structure des pores et les propriétés physiques des matériaux, reiation entre la structure des pores et la durabilité des matériaux.

\section{INFORMATIONS BIBLIOGRAPHIQUES}

\section{Concrete Technology \\ Vol. 2 - Practice}

de D.F. ORCHARD

Applied Science Publishers Londres.

Cette troisième édition du tome 2 d'une série de trois ouvrages consacrés à la technologie du béton traite principalement de la pratique du bétonnage. Il fait suite au tome 1 sur les propriétés des matériaux lui-même réédité très récemment. Rappelons que le tome 1 traitait principalement des ciments, des adjuvants, des granulats, du béton léger de la composition des bétons, des propriétés des liants et de la durabilité. L'auteur, D.F. Orchard est Professeur à l'Université de Sydney en Australie. Son ouvrage très bien documenté, ècrit en langue anglaise, comporte 440 pages dont 178 photocopies et 32 tableaux. Il est divisé en 11 chapitres précédés chacun d'un court résumé et se terminant par une bibliographie. Les principaux sujets abordés dans ces chapitres sont les suivants :

Chapitre 1 - Méthodes d'essais des ciments (prise et fausse prise, stabilité de volume, résistances mécaniques, finesse, composition chimique, chaleur d'hydratation).

Chapitre 2 - Essais sur béton frais (mesure de consistance et d'air occlus.
Chapitre 3 - Essais sur béton durci (résistances, module, abrasivité, perméabilité, retrait).

Chapitre 4 - Essais non destructifs.

Chapitre 5-Mise en place du béton (surtout à l'aide de la vibration).

Chapitre 6-Cure de béton (méthodes, effets de la cure ainsi que le traitement par la chaleur à la pression ordinaire et a pression élevée).

Chapitre 7 - Béton pompé (pompes et caractéristique du béton à utiliser).

Chapitre 8 - Les parements en béton et l'aspect de surface.

Chapitre 9 - Transport, manipulation et stockage des matériaux (ciments, granulats).

Chapitre 10 - Malaxage et mise en place du béton.

Chapitre 11 - Procédés (gunitage, vacuum concrete, injection).

Cet ouvrage est parfaitement à jour et l'auteur a employé le système d'unités international. Il sera profitable a tous les utilisateurs de béton connaissant l'anglais. Il constitue un ouvrage de référence très clair, suffisamment bien résumé mais où cependant le lecteur retrouvera l'essentiel des techniques modernes de bétonnage.
Introductory solid mechanics par James Mc. D. Baxter Brown Wiley, London

L'ouvrage traite en quinze chapitres de la théorie de l'élasticité et du calcul des structures. Diverses méthodes de résolution y sont décrites, en particulier la méthode des éléments finis pour les problèmes d'élasticité plane et la méthode des déformations pour les structures constituées par des barres à section constante.

On y trouve aussi l'examen de différents problèmes tels que l'élastoplasticité, et la charge critique d'instabilité de barres soumises à des conditions aux limites variées.

La lecture de cet ouvrage est simple à condition de connaître convenablement le calcul matriciel. Il constitue un excellent document de travail pour un ingénieur débutant ou désirant s'initier aux techniques modernes de calcul.

A signaler :

- Les toits plats et leur isolation thermique, première partie, note d'information technique 101 du Centre Scientifique $e_{t}^{t}$ Technique de la Construction.

- Compartamento sismico de edificios com estrutura parede, de A. Ravara, publication du Laboratorio Nacional de Engenharia Civil de Lisbonne, mémoire $n^{\circ} 423$.

- Steel box girder bridges, comptes rendus du colloque international organisé en février 1973 par l'Institution of Civil Engineers, Londres. 